\title{
Human Brain Dynamics Accompanying Use of Egocentric and Allocentric Reference Frames during Navigation
}

\author{
Klaus Gramann ${ }^{1,2}$, Julie Onton ${ }^{1}$, Davide Riccobon ${ }^{2}$, \\ Hermann J. Mueller ${ }^{2}$, Stanislav Bardins ${ }^{3}$, and Scott Makeig ${ }^{1}$
}

\begin{abstract}
Maintaining spatial orientation while travelling requires integrating spatial information encountered from an egocentric viewpoint with accumulated information represented within egocentric and/or allocentric reference frames. Here, we report changes in high-density electroencephalographic (EEG) activity during a virtual tunnel passage task in which subjects respond to a postnavigation homing challenge in distinctly different wayseither compatible with a continued experience of the virtual environment from a solely egocentric perspective or as if also maintaining their original entrance orientation, indicating use of a parallel allocentric reference frame. By spatially filtering the EEG data using independent component analysis, we found that these two equal subject subgroups exhibited differences in EEG power spectral modulation during tunnel passages in only a few
\end{abstract}

\section{INTRODUCTION}

The ability to build and maintain an accurate spatial representation of our environment is a prerequisite for maintaining spatial orientation, a complex task that involves use of distinct reference frames differing in their primitive parameters (Klatzky, 1998). Orienting using an egocentric reference frame integrates perceptual impressions gathered from a first-person perspective relative to the position and heading of the navigator. Orienting using an allocentric reference frame requires transformation of these egocentrically received impressions into a map-like representation incorporating angular and metric relationships that may be independent of the navigator's heading. During real-world navigation, information in both egocentric and allocentric reference frames may be integrated into a coherent representation of the environment, and the location and heading of the navigator within it (Bremmer, Schlack, Duhamel, Graf, \& Fink, 2001; Andersen, Snyder, Bradley, \& Xing, 1997). Predominant use of and reliance on one or the other of these reference frames can be influenced by the perspective from which the environment is experienced (e.g., from ground level or from an aerial viewpoint) (Shelton \& Gabrieli, 2002). In-

\footnotetext{
${ }^{1}$ University of California San Diego, La Jolla, CA, ${ }^{2}$ Ludwig-Maximilians University Munich, Germany, ${ }^{3}$ Klinikum der Universität München, Neurologische Klinik und Poliklinik, Munich, Germany
}

cortical areas. During tunnel turns, stronger alpha blocking occurred only in or near right primary visual cortex of subjects whose homing responses were compatible with continued use of an egocentric reference frame. In contrast, approaching and during tunnel turns, subjects who responded in a way compatible with use of an allocentric reference frame exhibited stronger alpha blocking of occipito-temporal, bilateral inferior parietal, and retrosplenial cortical areas, all areas implicated by hemodynamic imaging and neuropsychological observation in construction and maintenance of an allocentric reference frame. We conclude that in these subjects, stronger activation of retrosplenial and related cortical areas during turns support a continuous translation of egocentrically experienced visual flow into an allocentric model of their virtual position and movement.

dividual proclivities also strongly affect this weighting (Gramann, al Sharkawy, \& Deubel, in press; Bohbot, Lerch, Thorndycraft, Iaria, \& Zijdenbos, 2007; Etchamendy \& Bohbot, 2007; Gramann, Muller, Schonebeck, \& Debus, 2006; Gramann, Muller, Eick, \& Schonebeck, 2005).

The brain dynamics accompanying spatial orienting and navigation may be investigated using electroencephalographic (EEG) activity. Sufficient analyses of EEG data can reveal detailed information about macroscopic scale cortical processes across a wide range of time scales and frequencies. Invasive investigations of cortical field potentials during active spatial orienting in rodents typically reveal prominent modulations of $(4-12 \mathrm{~Hz})$ theta band power, demonstrating correlations between theta phase and the location of an animal in a place field (O'Keefe \& Burgess, 1999). The discovery of place cells in epileptic patients (Ekstrom et al., 2003) exhibiting similar dynamics in the human $(4-8 \mathrm{~Hz})$ theta band supports the assumption that allocentric representations are supported by human hippocampus. Reports that human theta band power recorded from the cortical surface is modulated during navigation in epileptic patients undergoing invasive monitoring (Caplan, Madsen, Raghavachari, \& Kahana, 2001; Kahana, Sekuler, Caplan, Kirschen, \& Madsen, 1999) motivated noninvasive EEG studies that also revealed increased theta activity during simulated navigation in healthy subjects (Bischof \& Boulanger, 2003; 
de Araujo, Baffa, \& Wakai, 2002). Moreover, modulation of alpha band (8-13 Hz) activity has been observed during navigation, dependent on the complexity of T-mazes (Caplan et al., 2001), and during route planning periods (de Araujo et al., 2002), with increases or decreases in alpha activity interpreted as damping of cortical excitability or increased readiness of cortical source domains to process incoming information, respectively (Pfurtscheller, 2001).

However, none of the studies examining spatial orienting have examined differences in brain dynamics dependent on the reference frames used by subjects during navigation. Previous work on spatial navigation suggests that subjects spontaneously adopt different strategies based on either an egocentric or an allocentric reference frame (Gramann et al., in press; Seubert, Humphreys, Muller, \& Gramann, 2008; Etchamendy \& Bohbot, 2007; Gramann et al., 2005, 2006; Bohbot, Iaria, \& Petrides, 2004). Here, we compare high-density EEG data recorded during simulated passages through virtual tunnels including a single bend to the left or to the right to study the brain dynamics patterns supporting subjects' proclivities to use different reference frames during navigation. Specifically, we investigated whether subject selection of an egocentric or an allocentric reference frame to choose their homing direction was associated with group differences in EEG brain dynamics on a subsecond scale, using spatial filtering of EEG data by independent component analysis (ICA) followed by time-frequency analysis.

\section{EXPERIMENTAL METHODS}

\section{Tunnel Task}

Previously, the first author and colleagues reported striking results from a virtual tunnel navigation task that produces sharp differences in the homing directions selected by subjects after passing through a virtual tunnel with one turn (Gramann et al., 2005, 2006). The tunnel simulation provides the navigator with sparse visual flow information on translational and rotational changes through the rate and direction of optic flow. During the passage, subjects are asked to keep track of their position with respect to their starting position. After a passage, subjects select or aim a screen image of a 3-D homing arrow so that it points back toward the implied virtual position of the tunnel entrance. A demonstration of the task is available on-line (sccn.ucsd.edu/ klaus/tunnel.html).

The surprising results of this behavioral test strongly suggest that normal adult subjects spontaneously adopt different spatial reference frames in determining the homing direction, possibly reflecting differences in their use of spatial reference frames during tunnel passages as well. Specifically, the response choices made by about half of all subjects tested are compatible with maintenance of their heading during the last tunnel segment. The responses of the other half of the subjects are com- patible with either a resumption or a continuation of the heading in which they first entered the tunnel. The choice of the first group is compatible with their predominant and continued use of an egocentric reference frame during the (egocentrically experienced) tunnel passages. The choice of the second group suggests that they also may build an allocentric model of the implied 3-D virtual environment during tunnel passages, a model in which they maintain their original heading, even when (e.g., during and after turns) this deviates from their perceived movement direction (Gramann et al., 2005).

In the present experiment, animations of passages through virtual tunnels consisting of five 3450-msec segments were shown by a projector (Sanyo PLC-XU47) above the observer's head on a $150 \mathrm{~cm} \times 150 \mathrm{~cm}$ white screen, occupying $41^{\circ}$ of their visual field. All tunnels began with two straight segments, followed by one turning segments to the left or right with different acute angled turns, and finally, two further straight segments.

\section{Subjects}

Thirty volunteer participants recruited from the academic community in Munich, Germany, took part in the experiment. Because of the imperfectly understood nature of sex differences in spatial navigation (Gron, Wunderlich, Spitzer, Tomczak, \& Riepe, 2000; Sandstrom, Kaufman, \& Huettel, 1998) and to increase statistical power, only male subjects were recruited. From a pool of 30 subjects, we selected for further testing 14 "nonturners," who preferentially used an allocentric reference frame in responding to the homing challenge, and 14 "turners," who preferentially used an egocentric reference frame to select their response (see Subject Characterization Experiment below). Of these, one subject had to be rejected from further analysis because of excessive EEG artifacts. All but three of the remaining 27 subjects (mean age = 24 years; standard deviation $=3.2$ years) were righthanded. None of the 27 had a history of neurological or psychiatric disorder, and each subject gave their informed consent to participate in the study, which was approved by the local human subject ethics committee.

\section{Subject Characterization Experiment}

In an initial subject characterization session, a week on average before the main experiment, subjects passively traversed tunnels with one turn of varying angle. Upon exiting each tunnel passage, two arrows were displayed and subjects had to decide which one of the displayed homing arrows pointed back to the origin of the traversed tunnel path. Each arrow represented the correct homing response within an egocentric or allocentric reference frame, respectively (see Figure $1 \mathrm{H}$ and $\mathrm{G}$ ). The two alternatives differed clearly in the first trials and then 


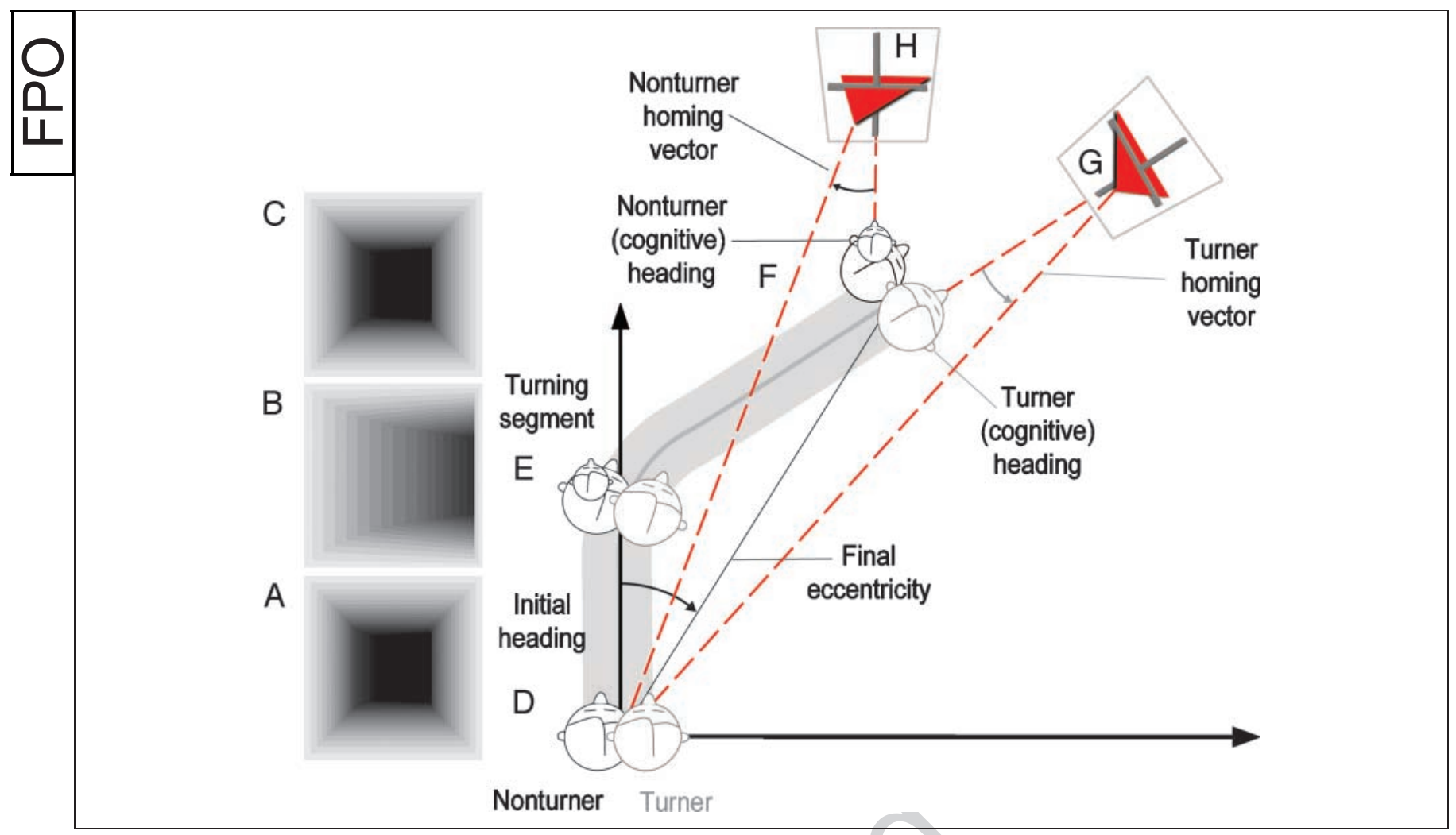

Figure 1. Schematic depiction of a passage through a tunnel with a turn to the right. The leftmost column displays snapshots of the navigator's view of (A) the first straightaway segment, (B) the following turn, here to the right, and (C) the final straightaway. Note that the illustration should not be considered correct with respect to the proportion of straight segments and the turning segment. The final eccentricity of this tunnel is about $40^{\circ}$ (as measured by arc DEF). The light gray heads (at D, E, and F) represent changes in the perceptual heading of turners as they pass through the virtual tunnel (gray background). For these subjects, the perceptual and cognitive headings remain identical. The adjacent black outline heads represent the headings of a nonturner, the larger head representing their perceptual heading and the smaller head, their cognitive heading during the first straightaway, the turn, and the final straightaway. Note that for nonturners the perceptual heading and the cognitive heading diverge during the turn (E). Panels $(\mathrm{G})$ and $(\mathrm{H})$ show sample homing arrows having the correct angular adjustment for the illustrated tunnel, (G) depicting the homing arrow judged correct by turners, and $(\mathrm{H})$ those judged correct by nonturners. Note that the homing arrow perceived as correct by turners (G) points behind and to the right of the navigator, whereas for nonturners the correct homing arrow $(\mathrm{H})$ points behind and to the left of the navigator. Remarkably, in experiments using the tunnel task, near equal numbers of subjects respond as turners and nonturners, respectively.

became increasingly difficult to discriminate in later trials. To be invited to take part in the main experiment, subjects had to select one or the other homing-vector solution in at least $75 \%$ of the trials, allowing them to be classified as turners or nonturners, respectively.

\section{Main Experiment}

The main experimental session comprised 120 tunnel trials. Of these, in 54 experimental trials, the tunnel consisted of five segments (each of 3.45-sec duration) with a turning middle segment. Turns produced varying final eccentricities of tunnel end position relative to the starting position $\left(15^{\circ}, 30^{\circ}\right.$, and $45^{\circ}$ to the left or to the right). Each eccentricity and direction combination was repeated nine times, in random order with slight variations in final eccentricity $\left(S D= \pm 1.33^{\circ}\right)$ to minimize any tendency toward categorical perception.

At the end of each passage, after leaving the tunnel, an image of a three-dimensional homing arrow was displayed with its direction adjusted to the correct answer for the participant's (turner or nonturner) response mode plus a small added angular deviation $\left( \pm 5^{\circ}, \pm 10^{\circ}\right)$. The subjects' task was to indicate whether the correct homing vector would point a bit more to the left or to the right, by pressing the respective left or right PC mouse button with their right index finger. The arrow disappeared after the mouse response. If there was no response by $3450 \mathrm{msec}$, the display went black and the next trial began.

Six filler trials were identical to the experimental trials except that their final tunnel eccentricities were between those of the 54 experimental trials. Filler trials were introduced to avoid forming categorical expectancies of possible end positions. Another 60 trials, serving as control trials, presented virtual passages through a tunnel consisting of two straight segments only. In these trials, subjects were instructed to watch the steady visual flow of the tunnel ribs, and to then press, at random, one of the two mouse buttons at the end of the passage upon display of the 3-D arrow. 


\section{Performance Measures and Statistics}

An initial series of ANOVAs was conducted to check for possible influences of the following factors on task performance: "direction" of the tunnel turn (left or right), "under/over"estimation of the homing angle, and magnitude of "error" $\left(5^{\circ}\right.$ or $\left.10^{\circ}\right)$. First, a mixed-design analysis with repeated measures over the factors "direction" (left, right) and final tunnel "eccentricity" $\left(15^{\circ}, 30^{\circ}\right.$, and $\left.45^{\circ}\right)$, with the subject "strategy" (turner or nonturner) as the between-subject measure, was computed. A second comparable analysis with repeated measures over the factors "under/over" and tunnel "eccentricity," with the factor "strategy" as between-subject measure, tested the influence of over- or underestimations of the displayed homing vector on the percentage of correct responses. Finally, the factors tunnel "direction" and arrow cue error "under/over" were collapsed in a final $2 \times 3$ mixed-design analysis with repeated measures over the factors cue angle "error" $\left(5^{\circ}\right.$ or $\left.10^{\circ}\right)$ and turn "eccentricity" $\left(15^{\circ}, 30^{\circ}\right.$, or $\left.45^{\circ}\right)$, with the subject's response "strategy" as between-subject measure.

\section{EEG Recording}

The EEG was recorded with an analog band pass from 0.016 to $100 \mathrm{~Hz}$ at a sampling rate of $500 \mathrm{~Hz}$, using $128 \mathrm{Ag} / \mathrm{AgCl}$ electrodes positioned according to the International 5\% system (Oostenveld \& Praamstra, 2001) referenced to Cz. Electrodes were contained in an elastic cap (Falk Minow Services, Munich, Germany); scalp impedance was brought below $10 \mathrm{~K} \Omega$. An additional electrode was placed on the infraorbital ridge of the left eye to record the vertical electrooculogram. Electrophysiological signals were amplified and recorded via BrainAmps (Brain Products, Munich, Germany). A 3-D position digitizing system (Zebris, CMS20S) was used to determine the positions on the scalp of the 128 electrodes.

\section{EEG Analyses}

More extensive descriptions of the EEG analysis methods can be found in the references listed in the Supplementary Notes. EEG data were re-referenced off-line to linked mastoids. Data intervals containing extreme peak-to-peak deflections or large bursts of electromyographic or other noise activity were excluded from further analysis. Eye movement artifacts did not qualify data for rejection. Data were analyzed by custom MATLAB scripts built on the open source EEGLAB toolbox (Delorme \& Makeig, 2004) (http://sccn.ucsd.edu/eeglab). After downsampling to $250 \mathrm{~Hz}$ and digitally filtering to remove frequencies above $50 \mathrm{~Hz}$, the data were submitted to extended infomax ICA (Lee, Girolami, \& Sejnowski, 1999) using the binica function (Makeig, Jung, Bell, Ghahremani, \& Sejnowski, 1997) from the EEGLAB toolbox. Default extended-mode binica training parameters were used with stopping weight change set to $1 e-7$.

\section{Component Selection}

Independent component (IC) activation time series for each subject were classified as brain activity or nonbrain artifacts by visual inspection of their activation spectra, time courses, and scalp topographies. An equivalent current dipole model was then computed for each brain IC scalp topography using a four-shell spherical head model implemented in the DIPFIT toolbox (Oostenveld \& Oostendorp, 2002). ICs with bilaterally distributed scalp maps were fit with a dual equivalent dipole model with a positional symmetry constraint. ICs with equivalent dipole models whose computed projection to the scalp electrodes accounted for less than 85\% of actual IC scalp map variance and ICs with model equivalent dipole(s) located outside the head sphere (e.g., those accounting primarily for scalp or neck muscle activities) were excluded from further analysis. This selection procedure produced an average of 19 localizable brain activity ICs per subject (range: 8 to 26), accounting, on average, for $80.16 \%$ (standard deviation $=11.51 \%$ ) of the EEG variance remaining after clear eye movement, electrocardiographic, and scalp muscle artifact ICs were removed from consideration.

\section{Component Power Spectra and Event-related Spectral Perturbation}

After decomposition, the data were separated into overlapping epochs of $4450 \mathrm{msec}$ (the duration of one tunnel segment, plus $500 \mathrm{msec}$ pre- and $500 \mathrm{msec}$ poststimulus time window). Each single-trial tunnel segment IC time series was transformed into a spectrographic image using three-cycle Morlet wavelets in a frequency range between 2 and $50 \mathrm{~Hz}$. Spectrographic images were composed into mean event-related spectral perturbation (ERSP) images by converting to log power, averaging trials for each segment, and then subtracting mean log power from a 3450-msec baseline interval drawn from the middle portion of straight control trial tunnels (Makeig, 1993). Concatenating the resulting ERSP images revealed mean event-related changes in spectral power in narrow-band frequency bins through the whole time course of tunnel passage. The log spectral baseline used for the arrow cue and response interval ERSPs was the ERSP for the arrow cue and random-response period in the control trials.

\section{Independent Component Clustering}

IC clustering across subjects was based on mean IC log spectra, event-related potentials (ERPs), scalp maps, equivalent dipole locations, ERSPs, and intertrial coherence for each selected IC from each subject. These measures were compressed by principal component analysis (PCA) into a single 25-dimensional cluster position vector for each IC after each measure (save dipole location) 
was first reduced to its 10 principal components (PCs). The equivalent dipole location measure was inherently three-dimensional but, to compensate, was multiplicatively weighted by a factor of 15 . ERSP PCs were weighted by 4; other measures (spectrum, ERP, intertrial coherence, and scalp topography) were given a weight of 1 . Finally, all this PCA-reduced joint measure information was combined into a single matrix of size 25 by ICs, whose dimensionality was then further reduced to 10 principal dimensions by PCA. ICs were then clustered using a $K$-means clustering algorithm applied to the matrix of IC-pair distances in this cluster position space, as implemented in EEGLAB. ICs whose distance to any cluster centroid in joint measure space was larger than three standard deviations from the mean were removed from the analysis. The number of clustered brain ICs in turners and nonturners (24.1 per subject in both groups) did not differ significantly $[F(1,25)=2.02, p>.167]$, nor did the number of ICs contributed by turners and nonturners to the 25 clusters [Group by Cluster interaction: $F(24,600)=$ $1.04, p>.410]$.

\section{ERSP Statistics}

Significant changes $(p<.001$, uncorrected for multiple comparisons) in mean power from the mean spectral baseline for each component over the time course of tunnel passages were computed using bootstrap resampling (Delorme \& Makeig, 2004). Nonsignificant time-frequency points were masked with zero values in the mean ERSPs. Grand means across subjects of these significance-masked ERSPs were then further masked for significance using a binomial test based on the computed permutation probability (with respect to $p=.001$ ) at each time-frequency point (Onton, Delorme, \& Makeig, 2005). To further avoid false positives from multiple comparisons, effects in the grand mean ERSPs that were significant at only a few neighboring voxels were not interpreted.

\section{Group-difference ERSP Computation}

To allow for detection of differences in ERSP features between turners and nonturners, it was necessary to frequencynormalize all subject IC spectrograms according to their respective mean peak alpha frequencies. This process was accomplished by linearly scaling individual IC frequencybin centers to set the mean peak alpha frequency of the baseline power spectrum (during control tunnel passages) to $10 \mathrm{~Hz}$. In this way, all peak alpha frequencies across components (with spectral peaks in the range $7.5-12 \mathrm{~Hz}$ ) were stretched/squeezed to a nominal $10 \mathrm{~Hz}$, and thus, any associated harmonics to $20 \mathrm{~Hz}, 30 \mathrm{~Hz}$, etc. The frequency-adjusted IC spectra were then reinterpolated to a common vector of nominal frequency bins between 3 and $45 \mathrm{~Hz}$.

Mean frequency-normalized IC ERSP images were created by subtracting the mean frequency-scaled baseline log spectrum from each latency of the mean ERSP image and similarly frequency-adjusted experimental tunnel segment spectrograms and then averaging over tunnel passages. For each IC cluster, frequency-adjusted subjectmean ERSP images were then averaged across cluster ICs separately for turners and nonturners, respectively. Permutation-based statistics for between-group ERSP differences were computed by first computing surrogate group means from sets of ERSPs drawn without substitution from ERSPs for the entire IC cluster in the same numbers in which turners or nonturners, respectively, contributed ICs to the cluster. Differences between such surrogate group-mean ERSPs formed a surrogate ERSP difference. At each time-frequency point, difference significance thresholds were taken to be 0.001 and 0.999 points in the distributions of 1500 such surrogate group-mean differences. Time-frequency points in the actual group-mean difference ERSPs were set to zero when their relative log power values did not exceed either $(p<.001)$ threshold. Only IC clusters that exhibited a significant difference for at least $1250 \mathrm{msec}$ were considered to show a reliable group difference.

\section{RESULTS}

\section{Behavioral Responses}

As expected from previous experiments, in all trials of the categorization task, the participants responded in a manner consistent either with their spontaneous adoption of a heading compatible with continued use during the traverse of an egocentric only or of an allocentric as well as an egocentric reference frame, respectively. Responses of 14 of the 27 participants, whose subsequently collected EEG data were suitable for analysis, were consistent with the predominant use of an egocentric reference frame. These turners (see grayed cartoon heads in Figure 1D-F) responded consistently with their having updated their cognitive heading aligned with their visually perceived heading change during the turn and then assumed that the homing arrow appeared before them in the heading in which they left the tunnel (Figure 1F). Following a rightward turn, therefore, these subjects indicated that the tunnel entrance (Figure 1D) was behind them and to their right (Figure 1G).

The other 13 participants in the EEG analysis (bold cartoon heads in Figure 1D-F) responded to the homing arrow in a way consistent with their imaging viewing the arrow appearing before them in the heading with which they first entered the tunnel. These "head nonturner" or "nonturner" subjects thus indicated that the entrance to a right turning tunnel was behind them and to their left (Figure $1 \mathrm{H}$ ). Their assumption that the homing arrows appeared in their original heading is consistent with the assumption that nonturners maintained a cognitive heading that, during tunnel turns and thereafter, was inconsistent with the egocentrically perceived visual flow, but 
consistent with an allocentric ("bird's-eye"-like) view of the tunnel environment, whose principal axis was aligned to the original heading with which they had entered the tunnel (Figure 1D).

Overall, participants made significantly more correct judgments $\left[F(1,25)=9.514, p<.005 ; \eta^{2}=.276\right]$ when the angle of the homing arrow differed from its veridical direction by $10^{\circ}$ as compared to $5^{\circ}$ (Figure 2), and for tunnels with the lowest $\left(15^{\circ}\right)$ eccentricity, with response accuracy decreasing monotonically as tunnel eccentricity increased $\left[F(2,50)=15.371, p<.001 ; \eta^{2}=.381\right]$. No further significant effects were observed. That turners and nonturners had near-identical response times [main effect group, $F(1,26)=2.934, p>.99$ ] suggests that nonturners developed an allocentric spatial representation as they passed through the simulated tunnels, rather than reconstructing it after leaving the tunnel.

\section{Common Brain Dynamics Accompanying Use of Egocentric and Allocentric Reference Frames}

Figure 3 presents equivalent dipole models and clustermean baseline spectra (left column), and event-related spectral dynamics (middle and right columns) for six representative clusters of IC processes whose scalp maps were well fit by a single equivalent dipole located within the brain volume (Makeig et al., 2002).

Baseline power spectra (Figure 3A, left panel) computed during passages through straight control tunnels included alpha band peaks (near $10 \mathrm{~Hz}$ ) for occipital

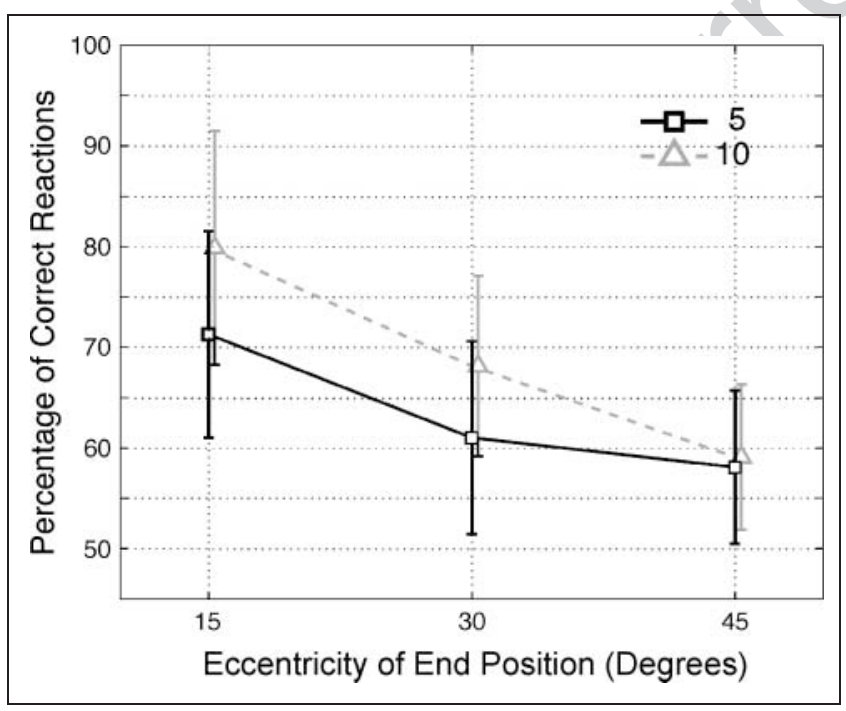

Figure 2. Percentage of correct reactions in the tunnel task as a function of eccentricity of end position $\left(15^{\circ}, 30^{\circ}\right.$, and $\left.45^{\circ}\right)$ relative to the starting point, and over- or underestimation of the displayed homing angle aggregated over left and right end positions and over- or underestimations of the displayed homing angle. The black solid line indicates $5^{\circ}$ deviations of the displayed homing angle as compared to the correct angular adjustment. The gray dotted line indicates $10^{\circ}$ deviations of the displayed homing angle as compared to the correct angular adjustment. and parietal cluster ICs (Figure 3B-D), whereas precentral cluster ICs (Figure 3E-F) exhibited peaks at mu activity frequencies (near 11 and $22 \mathrm{~Hz}$ ) and medial frontal IC spectra (Figure $3 \mathrm{G}$ ), a frontal-midline theta activity peak (near $6 \mathrm{~Hz}$ ). Figure 3A (middle and right columns) shows five screen shots of the tunnel animation captured at equally spaced intervals. Below them, mean ERSP images (Makeig, 1993) show log spectral power changes from baseline during the five corresponding tunnel segments (middle), and following appearance of the subsequently displayed 3-D arrow (right).

Upon tunnel entrance, a brief theta band power increase (warm colors) and significant intertrial coherence (indicating partially phase-locked theta activity and concomitant average ERP features, not shown) were followed by a 1-sec blocking of activity in the alpha band (near $10 \mathrm{~Hz}$ ) and at its first harmonic $(20 \mathrm{~Hz})$ in occipital and parietal component clusters (Figure 3B-D). When the upcoming tunnel turn first became visible (first dashed orange line), and throughout the turn (up to the second dash orange line), IC alpha activity in these clusters was again blocked relative to the baseline condition (i.e., while passing through the middle portions of straight control tunnels). Similar alpha blocking during turns occurred in all the IC clusters centered in parietal and occipital cortex (Clusters 15-18 and 20-25 in Supplementary Figures 1-6).

Following the turn, alpha activity (near $12 \mathrm{~Hz}$ ) above the baseline peak frequency $(10 \mathrm{~Hz})$ increased relative to the control tunnel baseline in the bilateral occipital cluster (Figure 3B). This pattern (alpha blocking followed by an upward shift in peak alpha frequency) reoccurred in both clusters as the tunnel exit (second red dashed line) came into view. The appearance of the 3-D arrow cue (right top) induced another brief (and partially phase-locked) theta complex followed, again, by alpha blocking accompanied by an increase in alpha band activity above the roughly 10-Hz peak. In the bilateral occipital cluster, this was followed by increased activity at the original alpha peak frequency. The spectral dynamics for a central superior parietal cluster (Figure 3D) were similar, though weaker (note difference in color scales), including alpha blocking at the peak alpha frequency (near $10 \mathrm{~Hz}$ ) and its first harmonic $(20 \mathrm{~Hz})$.

By contrast, in clusters in or near the precentral gyrus (BA 4), and more markedly on the right (Figure 3E), alpha/mu activity near 11 and $22 \mathrm{~Hz}$ (above the baseline peak frequency) increased, first at tunnel entrance, again more strongly during the turn, and finally, approaching and during tunnel exit. At the same time, low-frequency alpha band power near $9 \mathrm{~Hz}$ (and $18 \mathrm{~Hz}$ ) decreased slightly, this blocking intensifying in the left cluster (contralateral to the response hand; Figure $3 \mathrm{~F}$ ) as the tunnel exit approached. Following the appearance of the arrow response cue, baseline (near 9- $\mathrm{Hz}$ and $18-\mathrm{Hz}$ ) alpha activity in these clusters increased slightly following a brief $11-\mathrm{Hz}$ alpha power increase in the right cluster only. 


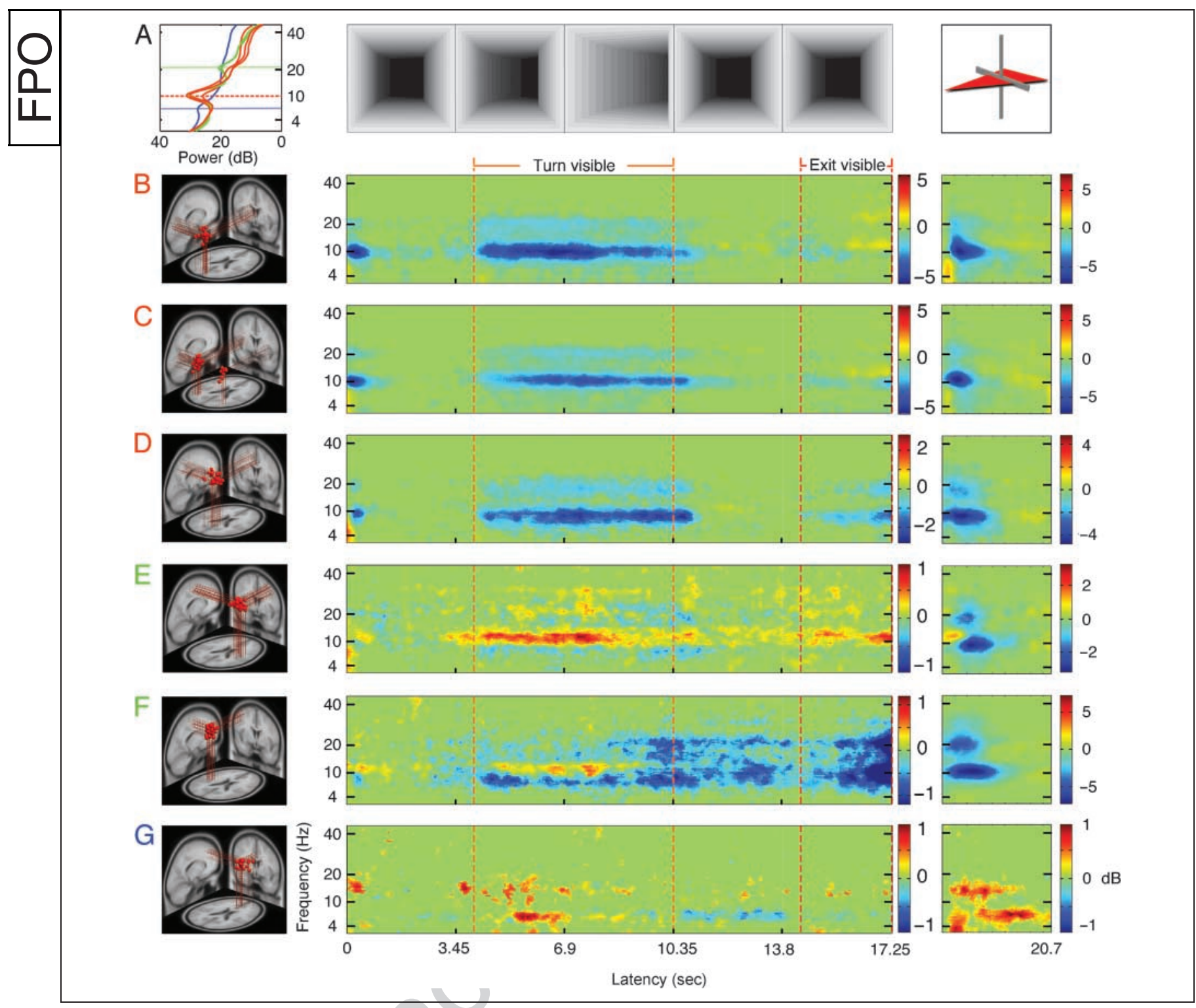

Figure 3. Mean ERSPs for selected IC clusters during tunnel passages. (A, left) Shows baseline mean log spectra during control trials removed from the ERSPs of six selected IC clusters $(B-G)$. Snapshots of a representative tunnel trial at five evenly spaced time points (spaced at intervals of $3450 \mathrm{msec}$ (A, middle) and at the appearance of the response prompt (A, right). (B-G, left) Locations of model equivalent dipoles for selected IC clusters, projected into a standard brain space, with each red sphere representing one cluster IC (or one of two bilaterally position-symmetric dipoles for cluster $\mathrm{C}$ ). (B-G, middle) Mean ERSP images for each of the IC clusters, revealing task-dependent changes in spectral power during navigation at log-spaced frequencies from 3 to $45 \mathrm{~Hz}$. Green indicates no significant difference in mean log power from baseline (visual stimulation during straight segments of the control trials). Other colors show significant deviations in log power (dB) from baseline (see color bars for scales). Vertical dashed orange lines indicate onset and offset of the period in which participants perceived the approaching and then (from 6.9 sec) currently occurring tunnel turn. Vertical dashed red lines indicate the period during which subjects saw the tunnel exit approaching. (B) IC cluster 23 (22 ICs from 12 turners and 9 nonturners), with the centroid located in or near right cuneus (BA 18; $x=-1, y=-79, z=7$ ); (C) IC cluster 21 (24 ICs from 11 turners, 8 nonturners), in or near the bilateral inferior occipital gyrus at the border to the temporal lobe (BA 19/37; $x=37, y=-70, z=-1$ ); (D) IC cluster 17 (26 ICs, 9 turners, 10 nonturners) in or near the precuneus (BA 7; $x=0, y=-45, z=43$ ); (E) IC cluster 12 ( 45 ICs, 12 turners, 11 nonturners) in or near the right precentral gyrus (BA 4; $x=36, y=-12, z=49$ ); (F) IC cluster 8 (26 ICs, 7 turners, 8 nonturners) in or near the left precentral gyrus (BA 4; $x=-40, y=-13, z=44$ ); (G) IC cluster 1 (24 ICs, 13 turners, 9 nonturners) in or near the right medial frontal gyrus (BA 9; $x=2, y=41, z=26$ ). See Supplemental Figures 1-6 for a description of all IC clusters.

Activity at the baseline peak theta frequency (near $6 \mathrm{~Hz}$ ) increased modestly as the turn approached in a medial frontal component cluster (Figure 3G) and then declined to slightly below baseline during the ensuing straightaway and tunnel exit. About $400 \mathrm{msec}$ after the appearance of the response arrow, a brief increase in low-theta power (strongest at $3 \mathrm{~Hz}$ or below) and a more sustained increase in low-beta (near $14 \mathrm{~Hz}$ ) activity appeared, followed by another weak but sustained theta band (near $6 \mathrm{~Hz}$ ) increase beginning near $1 \mathrm{sec}$ after cue appearance and continuing for 1 to $2 \mathrm{sec}$ after the mean response time (1123 msec). 


\section{EEG Differences between Strategy Groups}

The central question of the present investigation concerned brain dynamics accompanying the use of an allocentric or an egocentric reference frame to integrate perceived position and heading changes experienced during virtual navigation. Based on imaging studies and neuropsychological investigations, we expected differences in brain dynamics in cortical areas that are known to support use of egocentric and/or allocentric navigation strategies. In fact, just 5 of 30 derived IC clusters demonstrated pronounced differences in brain dynamics depending on the reference frame(s) used by the subject.

For an IC cluster located in or near the right cuneus (Figure 4B), alpha blocking when the upcoming turn became visible and then during the turn was significantly stronger for turners who responded using an egocentric reference frame. This alpha blocking was prolonged after the turn and more pronounced in turners when the end of the passage became visible. The right cuneus cluster was the only cluster with a significantly larger spectral per- turbation in turners. In contrast, nonturners using an allocentric reference frame to respond demonstrated significantly larger spectral perturbations in several frequency bands of four IC clusters located in or near the bilateral inferior occipital gyrus (Figure 4C), left and right inferior parietal cortex (Figure 4D and E), and retrosplenial cortex (Figure $4 \mathrm{~F}$ ), respectively. In the parietal and occipital IC clusters, alpha blocking at the peak alpha frequency during turns was more pronounced in nonturners; this stronger blocking also including the first alpha harmonic frequency. This was more pronounced in bilateral inferior occipital cortex than in more anterior clusters (as indicated by the scale bars in Figure 4).

The inferior occipital cluster ERSPs were significantly different for turners and nonturners, most pronounced approaching and during the turn itself; the left inferior parietal cluster exhibited prolonged alpha blocking following the turn, and the right inferior parietal component cluster exhibited stronger alpha blocking preceding the turn. Although the differences in left inferior parietal cortex did not reach significance for long after the turn, in right

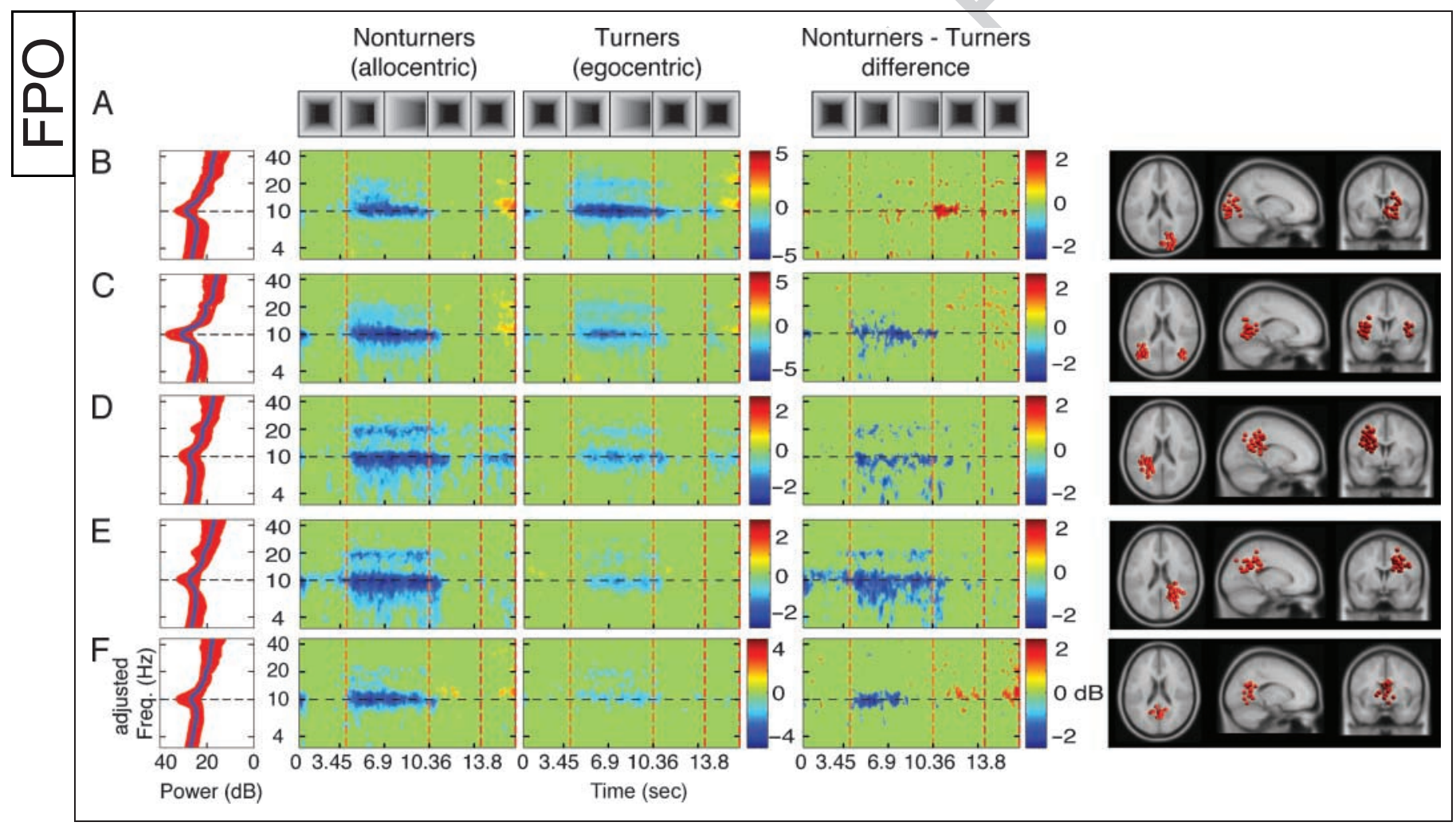

Figure 4. Component clusters revealing significant differences for the use of an egocentric or an allocentric reference frame during spatial navigation. Top row (A) displays the time course of a representative tunnel trial with five segments for nonturners, using an allocentric reference frame, and for turners, using an egocentric reference frame, and for the difference ERSPs (computed by subtracting ERSP activity of turners from ERSP activity or nonturners). Rows B to F display from left to rightmost column frequency-rescaled (i) baseline power spectrum of control trials, (ii) ERSP activity for nonturners, (iii) ERSP activity for turners, (iv) difference ERSPs computed by subtracting ERSP activity of turners from ERSP activity of nonturners, and (v) cluster IC equivalent dipoles projected on horizontal, sagittal, and coronal view of the standard brain for (B) a component cluster with the cluster centroid located in or near the right inferior occipital gyrus $(x=-37, y=-70, z=-1)$, (C) a component cluster in or near the bilateral inferior occipital gyrus at the border to the temporal lobe (BA 19/37; $x=37, y=-67, z=-1$ ), (D) a component cluster located in or near the left inferior parietal lobule (BA 40; $x=-30, y=-40, z=33$ ), (E) a component cluster located in or near the right precuneus $(x=26, y=-47, z=32)$, and $(\mathrm{F})$ a component cluster located in or near retrosplenial cortex at the posterior pole of cingulate cortex $(x=1, y=-56, z=10)$. Color coding and dashed vertical lines as in Figure 2 . 


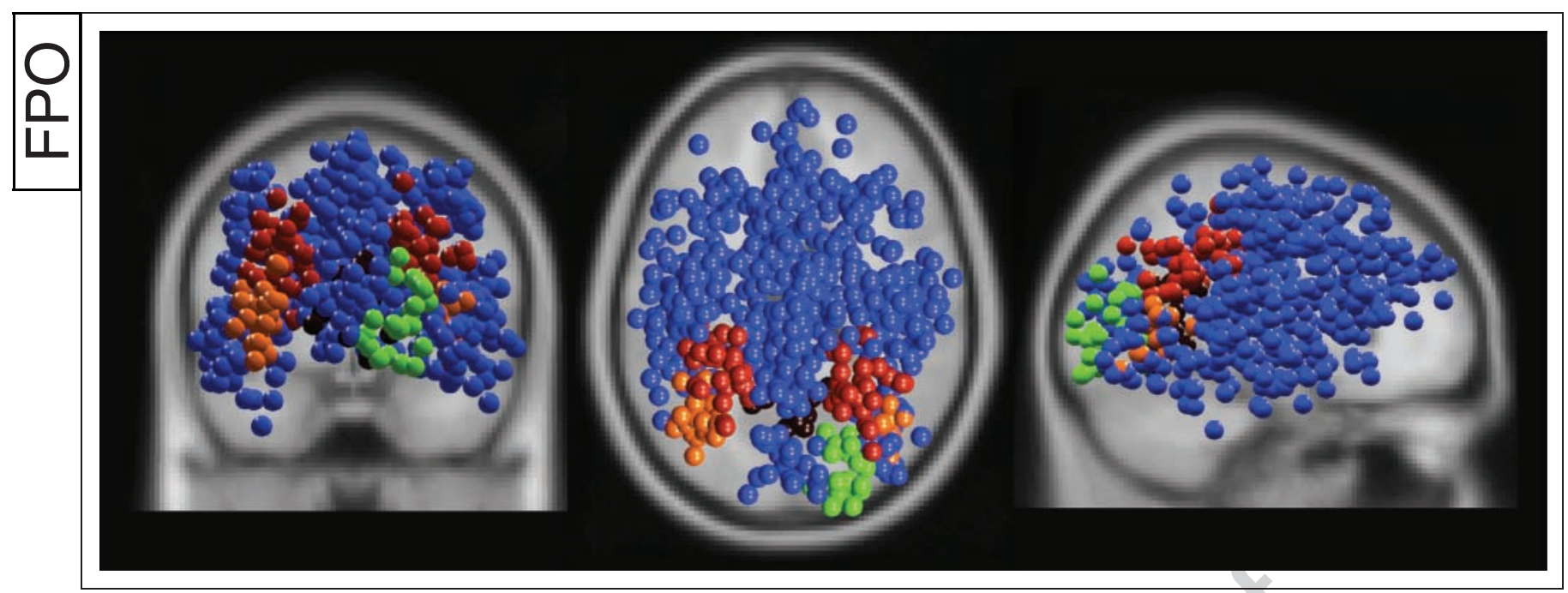

Figure 5. Equivalent model dipole locations for IC clusters exhibiting and not-exhibiting ERSP differences between turner and nonturner subject groups. Blue balls show equivalent model dipole locations of IC processes in 25 clusters without significant ERSP group differences; (green) cluster in or near the right cuneus (Figure 4B); (orange) cluster in or near the bilateral inferior occipital gyrus (Figure 4C); (red) cluster in or near left and right inferior parietal cortex (Figure 4D and E); (dark red) cluster in or near medial inferior retrosplenial cortex (Figure 4F).

inferior parietal cortex, alpha blocking was significantly deeper in nonturners from the very beginning of the tunnel passage.

Finally, alpha blocking (or "desynchronization") in or near retrosplenial cortex was significantly stronger approaching and during the turn for nonturners as compared to turners. Retrosplenial cluster alpha blocking was confined to the first half of the turning segment. In addition, the retrosplenial cluster was the only cluster in which upper-alpha activity was stronger in nonturner subjects, first after the turn and again as the exit approached.

Equivalent dipole locations for ICs in the five clusters with significant ERSP group differences are shown in various colors in Figure 5; equivalent dipole locations for ICs in the 25 clusters without subject group differences are colored blue. Note the relative regional specificity of the processes associated with strategy group differences.

\section{DISCUSSION}

Here we report task-strategy dependent differences in spatially resolved EEG brain dynamics in a relatively wide (3-30 Hz) frequency range during path integration in virtual navigation. To our knowledge, this is the first study to investigate differences in EEG dynamics associated with individual differences in the use of spatial reference frames. As expected, group differences in the preferred reference frame used by subjects to select homing responses after navigation were, indeed, associated with group differences in EEG dynamics during the navigation. However, although separable clusters of independent EEG signals during spatial navigation were recorded from more than 30 cortical areas, only a small subset of these distinguished the two subject groups using an egocentric or an allocentric reference frame to build a spatial representa- tion of the virtual environment during passive navigation and then to select a homing direction after tunnel egress. Notably, the brain areas exhibiting the group differences are areas implicated in construction of an allocentric model of the environment by neuropsychological and hemodynamic brain imaging studies.

\section{EEG Spatial Resolution and ICA}

Unlike hemodynamic imaging methods, high-density EEGbased functional brain imaging using ICA spatial filtering allows measurement of mean changes in cortical dynamics with high temporal and spectral resolution, while giving spatial resolution at least sufficient, in our data, to distinguish 30 spatially separable clusters of brain EEG sources across our subject population, plus several distinct classes of nonbrain artifacts. The association between component (IC) processes with maximally independent time courses and "dipolar" scalp maps is not yet widely understood.

In general, as is well known, the EEG inverse problem of determining the source distribution giving rise to a given scalp potential distribution is not only technically challenging but also impossible without further constraints, as any number of self-canceling source configurations can be added to an inverse solution to give another equally exact solution. However, the inverse problem of determining the origin of a single current dipole, given a dipolar scalp map, is not underdetermined. Current dipoles are useful for EEG source imaging because, by biophysics, synchronous source activity across a closed cortical patch will have a projection to the scalp matching that of a nearby dipole termed its equivalent dipole model (Scherg, 1990). Because source localization of EEG data using equivalent current dipoles gives only an approximation of the actual locations of the cortical source patches, in this report, 
we refer to IC brain processes as occurring "in or near" their equivalent dipole model locations.

The abundant occurrence (as in these data) of EEG processes with (maximally) independent time courses and (maximally) dipolar scalp maps (Delorme et al., in preparation; Makeig et al., 2004) arises from the connectivity structure of cortex, which highly favors short-range connections, particularly of inhibitory neuronal networks, and predominantly radial, spatially segregated thalamocortical connections. These cause emergent local field activity to spread near-synchronously (for yet unknown reasons) through a connected cortical domain or patch, a phenomenon termed by Freeman and Barrie (2000) "phase cones," producing far-field potentials that sum at the scalp surface with other, independently generated source signals, and are recorded as EEG signals. Occasionally, independent component processes sum synchronous activities in two cortical patches whose field activities become tightly coupled by direct fiber tract pathways (e.g., corpus callosum) or common inputs.

The association of ICs and dipolar scalp maps greatly simplifies the problem of finding spatially "simple" maps representing the projection of only one (or occasionally two) localizable EEG source area(s). The dominant approach to the problem of finding "simple" scalp maps has been to select scalp maps occurring at peak excursions in average (ERP) waveforms, though after about $30 \mathrm{msec}$, ERPs rarely sum potentials from only one source area (Hupe et al., 2001). Finding the actual cortical patch, given its (nearby) equivalent dipole location, is a more technically challenging problem. However, recent results (Wipf \& Nagarajan, 2009) suggest that estimates of the actual cortical source patches supporting IC processes may soon become available for EEG studies that also record subject magnetic resonance head images, allowing construction of individual electrical head models (Akalin Acar \& Makeig, 2008).

\section{First-person EEG Brain Dynamics}

Both response groups in this experiment necessarily perceived visual translation and rotation information from the same first-person perspective. Compatible with functional imaging studies reporting increased hemodynamic activity in parietal and occipital areas during navigation (Wolbers, Wiener, Mallot, \& Buchel, 2007; Wolbers \& Buchel, 2005; Committeri et al., 2004; Hartley, Maguire, Spiers, \& Burgess, 2003; Iaria, Petrides, Dagher, Pike, \& Bohbot, 2003; Shelton \& Gabrieli, 2002; Maguire et al., 1998), this visuospatial processing was accompanied by widespread alpha blocking in occipital and parietal cortex. Alpha blocking or "desynchronization" in visual cortical areas likely reflects enhanced visual attention and cortical excitability during processing of changing visual flow information at tunnel entrances, during and approaching tunnel turns, and at tunnel exits (Klimesch, Sauseng, \& Hanslmayr, 2007; Worden, Foxe, Wang, \& Simpson, 2000). Because of the power of ICA to separate ongoing cortical source activities, this may be the first article to spatially separate the dynamics of alpha band activities in 20 or more cortical regions from noninvasively recorded EEG data. A general inverse relationship between concurrent alpha band EEG power and BOLD signal has also been reported by several studies (Laufs et al., 2006; Goldman, Stern, Engel, \& Cohen, 2002). However, differences in more anterior brain regions (e.g., parietal, motor, and premotor cortex) are difficult to explain as linked to perception of visual flow and can be assumed to reflect brain dynamics accompanying higher cognitive functions supporting spatial orientation, path integration, and other aspects of task performance.

The observed activation pattern in or near superior parietal cortex (Figure 3D) is consonant with the wellsupported role for this region in heading estimation and spatial orienting (Hartley et al., 2003; Vogeley \& Fink, 2003; Peuskens, Sunaert, Dupont, Van Hecke, \& Orban, 2001; Maguire et al., 1998). The parallel alpha-blocking pattern for the parietal midline IC cluster (Figure 3B) resembled those for occipital clusters (Figure 3C, Supplementary figures), consistent with the model of optic flow as activating the dorsal cuneus, which then distributes information to the dorsal parietal and occipito-temporal areas that process different aspects of visual flow (de Jong, Shipp, Skidmore, Frackowiak, \& Zeki, 1994). Thus, although occipito-temporal alpha blocking (Figure 4B) may index the major role of MT+ in movement processing and estimation of momentary heading direction from visual flow (Wolbers et al., 2007; Goossens, Dukelow, Menon, Vilis, \& van den Berg, 2006; Peuskens et al., 2001; Morrone et al., 2000; de Jong et al., 1994), superior parietal alpha blocking (Figure 3B) might reflect processing of upcoming path information to anticipate future heading changes (Field, Wilkie, \& Wann, 2007).

Lower-frequency alpha band and beta band activity in the left hemisphere IC cluster in or near premotor cortex and contralateral to the right-hand subject button presses was also blocked before and during voluntary hand movements (Figure 3F), mimicking typical motor-related mu blocking over the Rolandic regions (Pfurtscheller \& Aranibar, 1979). An activity increase that is stronger in the (ipsilateral) right cluster (Figure 3E), near $12 \mathrm{~Hz}$ during turns and again before button presses, has not been reported, to our knowledge, in studies of motor-related activity, and might conceivably index activity related to saccadic and optokinetic eye movements during visual flow stimulation (Dieterich, Bense, Stephan, Yousry, \& Brandt, 2003; Petit \& Haxby, 1999; Brandt, Bartenstein, Janek, \& Dieterich, 1998).

Finally, mean theta power increases in or near medial frontal cortex before and during tunnel turns (Figure 3G) likely reflect increased workload associated with spatial integration during the turns. Medial frontal cortex at the level of the dorsal anterior cingulate also shows taskdependent theta power increases during increased memory demands (Onton et al., 2005) and is active during virtual navigation tasks (Wolbers et al., 2007; Hartley et al., 
2003; Iaria et al., 2003; Shelton \& Gabrieli, 2002; Maguire et al., 1998), possibly reflecting the engagement of processes involving active spatial working memory. The absence, in our data, of sustained cortical theta activity peaks in medial temporal and other cortical areas is possibly related to the "passive" quality of the navigation in this task (i.e., it involved no route planning), to the absence of landmarks in the virtual environments, and/or to the simple path layouts in this task compared to studies reporting cortical theta activity during navigation (Caplan et al., 2003; de Araujo et al., 2002; Kahana et al., 1999; O’Keefe $\&$ Burgess, 1999). Note that there are, to date, no reports of scalp-recorded EEG activity sources in medial temporal areas in which theta phenomena related to navigation might be most expected. Documenting medial temporal sources of scalp EEG signals would be difficult, as it would require ruling out possible adjacent cortical source locations.

\section{Reference Frame-dependent EEG Dynamics}

Our results demonstrate that the computation and maintenance of a parallel allocentric reference frame by about half of the subjects during navigation was accompanied by increased EEG activation in a distributed set of brain areas, affecting a broad EEG frequency range (at least 3-22 Hz). Following the tunnel turn, turners who performed path integration within an egocentric reference frame produced stronger alpha desynchronization of IC EEG sources in or near the right inferior occipital gyrus (Figure 4B) following the tunnel turn. By contrast, nonturners who maintained an allocentric reference frame during navigation exhibited stronger alpha blocking preceding and during heading changes in or near bilateral occipito-temporal cortex (Figure 4C). This difference in alpha blocking in or near V3 and MT+ before, during, and after tunnel turns is consistent with an allocentric strategy-specific increase in attention to the information processed in these areas (Klimesch et al., 2007; Worden et al., 2000).

The strategy-specific attention-related activation increase in parietal (Figure 4D-F) cortex areas might also reflect use of visual imagery by those subjects using an allocentric reference frame. Increased activation in bilateral parietal cortex was reported for object-centered (vs. ego-centered) allocation of attention (Wilson, Woldorff, \& Mangun, 2005), supporting the assumption that nonturners may imagine the tunnel passage from a straight-ahead or bird's-eye view. Comparable forms of perspective changes can be seen in research on embodiment. Imaging egocentric information from a perspective other than provided by the actual physical body perspective (disembodiment) is accompanied by increased activation near the temporo-parietal junction (Arzy, Thut, Mohr, Michel, \& Blanke, 2006; Blanke, Mohr, Michel, Pascual-Leone, \& Thut, 2005; Blanke, Mohr, Michel, Pascual-Leone, Brugger, et al., 2005). Thus, the dynamic group differences, here, in bilateral inferior parietal cortex (Figure 4D and E) might index a more object-based, dis- embodied processing of egocentric visual flow in the nonturner group, although we did not debrief subjects about their use of visual imagery and so have no direct evidence of such as association.

Note that these EEG group differences cannot be explained by strategy-specific differences in eye movements because spectral dynamics of ICs accounting for artifacts produced by horizontal and vertical eye movements in both strategy groups were comparable (see Supplementary Figure 6, and also Gramann et al., in press). Further, eye movement ICs did not project strongly to parietal sites, did not noticeably influence the nearly independent posterior IC activities, and exhibited no perturbations in alpha band power during tunnel passages. Thus, we conclude that individual differences in visuospatial information processing involving use of different reference frame strategies during path integration are associated with differences in EEG dynamics in early visual areas (Figure 4B and $\mathrm{C}$ ) that inform higher stages of visual motion processing and spatial orientation (Figure 4D-F). However, here we did not look for fine temporal connections between eye movements and posterior alpha activities, which might exist because of their common association with visual attention.

Strategy-specific brain dynamics were also observed in inferior parietal cortex, most pronounced in or near the right precuneus (Figure 4E). Posteromedial parietal cortex has reciprocal cortico-cortical connections with retrosplenial cortex (Cavanna \& Trimble, 2006) and is assumed to elaborate egocentric and allocentric spatial relationships in concert with lateral parietal areas. Nonturners exhibited stronger alpha blocking approaching and during turns, when updating of heading changes within visual egocentered and computed allocentric reference frames was most demanding. The functional significance of the additional blocking of low alpha band activity in nonturners is unknown, but is at least compatible with the assumption that the precuneus plays a major role in building and/or maintaining concurrent egocentric and allocentric reference frame representations that support the use of optic flow information in guiding self-motion (de Jong et al., 1994).

Importantly, the spontaneous maintenance of an allocentric reference frame in nonturners was also associated with stronger alpha desynchronization in or near retrosplenial cortex, a cortical region implicated in translating between reference frames (Whitlock, Sutherland, Witter, Moser, \& Moser, 2008; Byrne, Becker, \& Burgess, 2007; Wolbers \& Buchel, 2005; Maguire, 2001). Here, we demonstrated that construction and maintenance of an allocentric reference frame is associated with stronger alpha blocking in or near retrosplenial cortex preceding and during heading changes (Figure 4F), when the cognitive and the current perceived heading diverge. This phenomenon likely reflects the transformation of the initial egocentrically perceived spatial information into an allocentric reference frame. The observed upper alpha band synchronization 
(near $12 \mathrm{~Hz}$ ) in retrosplenial cortex following heading changes (Figure $4 \mathrm{~F}$, right) might reflect the inhibition of processing further visuospatial information from a firstperson perspective to avoid interference with maintaining two spatial reference frames (Klimesch, Doppelmayr, Schwaiger, Auinger, \& Winkler, 1999). However, the functional role or roles of alpha frequency modulation within EEG source processes is not yet clear.

In summary, our results demonstrate that advanced EEG-based functional brain imaging using ICA-derived spatial filtering can be used to study network dynamics of spatial orienting and navigation. Using data-driven ICA spatial filtering on high-density EEG data and equivalent dipole modeling of IC source locations, we were able to describe the task- and strategy-dependent modulation of several frequency bands underlying computation and maintenance of distinct reference frames on a subsecond time scale. Also, our results show that future behavioral and brain imaging studies of human navigation should take into account individual subject differences in navigation approach and strategy. Our results support our conclusion that preferred use of an egocentric or an allocentric reference frame during spatial navigation is accompanied by differences in EEG brain dynamics in cortical areas involved in integrating visual flow information with changes in cognitive heading, and in areas associated with the transfer of egocentrically experienced spatial information into an allocentric reference frame.

\section{Acknowledgments}

This research was supported by a grant from the German Research Foundation (DFG GR2627/2-1), a research stipend from the G.A. Lienert Foundation for K. G., a gift from the Swartz Foundation (Old Field, NY), and by grants from the CalIT2 Institute, UCSD and from the National Science Foundation, USA (NSF IIS-0613595, TDLC-0542013)

Reprint requests should be sent to Klaus Gramann, Swartz Center for Computational Neuroscience, Institute for Neural Computation, University of California San Diego, La Jolla, CA 92093-0961, orvia e-mail:klaus@sccn.ucsd.edu.

\section{REFERENCES}

Akalin Acar, Z., \& Makeig, S. (2008). Neuroelectromagnetic forward modeling toolbox. Conference Proceedings of the IEEE Engineering in Medicine and Biology Society, 2008, 3991-3994.

Andersen, R. A., Snyder, L. H., Bradley, D. C., \& Xing, J. (1997). Multimodal representation of space in the posterior parietal cortex and its use in planning movements. Annual Review of Neuroscience, 20, 303-330.

Arzy, S., Thut, G., Mohr, C., Michel, C. M., \& Blanke, O. (2006). Neural basis of embodiment: Distinct contributions of temporoparietal junction and extrastriate body area. Journal of Neuroscience, 26, 8074-8081.

Bischof, W. F., \& Boulanger, P. (2003). Spatial navigation in virtual reality environments: An EEG analysis. CyberPsychology \& Behavior, 6, 487-495.
Blanke, O., Mohr, C., Michel, C., Pascual-Leone, A., \& Thut, G. (2005). Linking the out-of-body experience to self processing at the temporo-parietal junction. Journal of Cognitive Neuroscience, 23-24.

Blanke, O., Mohr, C., Michel, C. M., Pascual-Leone, A., Brugger, P., Seeck, M., et al. (2005). Linking out-of-body experience and self processing to mental own-body imagery at the temporoparietal junction. Journal of Neuroscience, 25, 550-557.

Bohbot, V. D., Iaria, G., \& Petrides, M. (2004). Hippocampal function and spatial memory: Evidence from functional neuroimaging in healthy participants and performance of patients with medial temporal lobe resections. Neuropsychology, 18, 418-425.

Bohbot, V. D., Lerch, J., Thorndycraft, B., Iaria, G., \& Zijdenbos, A. P. (2007). Gray matter differences correlate with spontaneous strategies in a human virtual navigation task. Journal of Neuroscience, 27, 10078-10083.

Brandt, T., Bartenstein, P., Janek, A., \& Dieterich, M. (1998). Reciprocal inhibitory visual-vestibular interaction. Visual motion stimulation deactivates the parieto-insular vestibular cortex. Brain, 121, 1749-1758.

Bremmer, F., Schlack, A., Duhamel, J. R., Graf, W., \& Fink, G. R. (2001). Space coding in primate posterior parietal cortex. Neuroimage, 14, S46-S51.

Byrne, P., Becker, S., \& Burgess, N. (2007). Remembering the past and imagining the future: A neural model of spatial memory and imagery. Psychological Review, 114, 340-375.

Caplan, J. B., Madsen, J. R., Raghavachari, S., \& Kahana, M. J. (2001). Distinct patterns of brain oscillations underlie two basic parameters of human maze learning. Journal of Neurophysiology, 86, 368-380.

Caplan, J. B., Madsen, J. R., Schulze-Bonhage, A., Aschenbrenner-Scheibe, R., Newman, E. L., \& Kahana, M. J. (2003). Human theta oscillations related to sensorimotor integration and spatial learning. Journal of Neuroscience, 23, 4726-4736.

Cavanna, A. E., \& Trimble, M. R. (2006). The precuneus: A review of its functional anatomy and behavioural correlates. Brain, 129, 564-583.

Committeri, G., Galati, G., Paradis, A. L., Pizzamiglio, L., Berthoz, A., \& LeBihan, D. (2004). Reference frames for spatial cognition: Different brain areas are involved in viewer-, object-, and landmark-centered judgments about object location. Journal of Cognitive Neuroscience, 16, $1517-1535$.

de Araujo, D. B., Baffa, O., \& Wakai, R. T. (2002).

Theta oscillations and human navigation: A magnetoencephalography study. Journal of Cognitive Neuroscience, 14, 70-78.

de Jong, B. M., Shipp, S., Skidmore, B., Frackowiak, R. S., \& Zeki, S. (1994). The cerebral activity related to the visual perception of forward motion in depth. Brain, 117, 1039-1054.

Delorme, A., \& Makeig, S. (2004). EEGLAB: An open source toolbox for analysis of single-trial EEG dynamics including independent component analysis. Journal of Neuroscience Methods, 134, 9-21.

Dieterich, M., Bense, S., Stephan, T., Yousry, T. A., \& Brandt, T. (2003). fMRI signal increases and decreases in cortical areas during small-field optokinetic stimulation and central fixation. Experimental Brain Research, 148, $117-127$.

Ekstrom, A. D., Kahana, M. J., Caplan, J. B., Fields, T. A., Isham, E. A., Newman, E. L., et al. (2003). Cellular networks underlying human spatial navigation. Nature, 425, 184-188. 
Etchamendy, N., \& Bohbot, V. D. (2007). Spontaneous navigational strategies and performance in the virtual town. Hippocampus, 17, 595-599.

Field, D. T., Wilkie, R. M., \& Wann, J. P. (2007). Neural systems in the visual control of steering. Journal of Neuroscience, 27, 8002-8010.

Freeman, W. J., \& Barrie, J. M. (2000). Analysis of spatial patterns of phase in neocortical gamma EEGs in rabbit. Journal of Neurophysiology, 84, 1266-1278.

Goldman, R. I., Stern, J. M., Engel, J., Jr., \& Cohen, M. S. (2002). Simultaneous EEG and fMRI of the alpha rhythm. NeuroReport, 13, 2487-2492.

Goossens, J., Dukelow, S. P., Menon, R. S., Vilis, T., \& van den Berg, A. V. (2006). Representation of head-centric flow in the human motion complex. Journal of Neuroscience, 26, 5616-5627.

Gramann, K., al Sharkawy, J., \& Deubel, H. (in press). Eye movements during virtual navigation. International Journal of Neuroscience.

Gramann, K., Muller, H. J., Eick, E. M., \& Schonebeck, B. (2005). Evidence of separable spatial representations in a virtual navigation task. Journal of Experimental Psychology: Human Perception and Performance, 31, 1199-1223.

Gramann, K., Muller, H. J., Schonebeck, B., \& Debus, G. (2006). The neural basis of ego- and allocentric reference frames in spatial navigation: Evidence from spatio-temporal coupled current density reconstruction. Brain Research, $1118,116-129$.

Gron, G., Wunderlich, A. P., Spitzer, M., Tomczak, R., \& Riepe, M. W. (2000). Brain activation during human navigation: Gender-different neural networks as substrate of performance. Nature Neuroscience, 3, 404-408.

Hartley, T., Maguire, E. A., Spiers, H. J., \& Burgess, N. (2003). The well-worn route and the path less traveled: Distinct neural bases of route following and wayfinding in humans. Neuron, 37, 877-888.

Hupe, J. M., James, A. C., Girard, P., Lomber, S. G., Payne, B. R., \& Bullier, J. (2001). Feedback connections act on the early part of the responses in monkey visual cortex. Journal of Neurophysiology, 85, 134-145.

Iaria, G., Petrides, M., Dagher, A., Pike, B., \& Bohbot, V. D. (2003). Cognitive strategies dependent on the hippocampus and caudate nucleus in human navigation: Variability and change with practice. Journal of Neuroscience, 23, 5945-5952.

Kahana, M. J., Sekuler, R., Caplan, J. B., Kirschen, M., \& Madsen, J. R. (1999). Human theta oscillations exhibit task dependence during virtual maze navigation. Nature, 399, 781-784.

Klatzky, R. L. (1998). Allocentric and egocentric spatial representations: Definitions, distinctions, and interconnections (Vol. 1). New York: Springer.

Klimesch, W., Doppelmayr, M., Schwaiger, J., Auinger, P., \& Winkler, T. (1999). "Paradoxical" alpha synchronization in a memory task. Brain Research, Cognitive Brain Research, 7, 493-501.

Klimesch, W., Sauseng, P., \& Hanslmayr, S. (2007). EEG alpha oscillations: The inhibition-timing hypothesis. Brain Research Reviews, 53, 63-88.

Laufs, H., Holt, J. L., Elfont, R., Krams, M., Paul, J. S., Krakow, K., et al. (2006). Where the BOLD signal goes when alpha EEG leaves. Neuroimage, 31, 1408-1418.

Lee, T. W., Girolami, M., \& Sejnowski, T. J. (1999). Independent component analysis using an extended infomax algorithm for mixed subgaussian and supergaussian sources. Neural Computation, 11, 417-441.
Maguire, E. A. (2001). The retrosplenial contribution to human navigation: A review of lesion and neuroimaging findings. Scandinavian Journal of Psychology, 42, 225-238.

Maguire, E. A., Burgess, N., Donnett, J. G., Frackowiak, R. S., Frith, C. D., \& O'Keefe, J. (1998). Knowing where and getting there: A human navigation network. Science, 280, 921-924.

Makeig, S. (1993). Auditory event-related dynamics of the EEG spectrum and effects of exposure to tones. Electroencephalography and Clinical Neurophysiology, 86, 283-293.

Makeig, S., Jung, T. P., Bell, A. J., Ghahremani, D., \& Sejnowski, T. J. (1997). Blind separation of auditory event-related brain responses into independent components. Proceedings of the National Academy of Sciences, U.S.A., 94, 10979-10984.

Makeig, S., Westerfield, M., Jung, T. P., Enghoff, S., Townsend, J., Courchesne, E., et al. (2002). Dynamic brain sources of visual evoked responses. Science, 295, 690-694.

Morrone, M. C., Tosetti, M., Montanaro, D., Fiorentini, A., Cioni, G., \& Burr, D. C. (2000). A cortical area that responds specifically to optic flow, revealed by fMRI. Nature Neuroscience, 3, 1322-1328.

O'Keefe, J., \& Burgess, N. (1999). Theta activity, virtual navigation and the human hippocampus. Trends in Cognitive Sciences, 3, 403-406.

Onton, J., Delorme, A., \& Makeig, S. (2005). Frontal midline EEG dynamics during working memory. Neuroimage, 27, 341-356.

Oostenveld, R., \& Oostendorp, T. F. (2002). Validating the boundary element method for forward and inverse EEG computations in the presence of a hole in the skull. Human Brain Mapping, 17, 179-192.

Oostenveld, R., \& Praamstra, P. (2001). The five percent electrode system for high-resolution EEG and ERP measurements. Clinical Neurophysiology, 112, 713-719.

Petit, L., \& Haxby, J. V. (1999). Functional anatomy of pursuit eye movements in humans as revealed by fMRI. Journal of Neurophysiology, 82, 463-471.

Peuskens, H., Sunaert, S., Dupont, P., Van Hecke, P., \& Orban, G. A. (2001). Human brain regions involved in heading estimation. Journal of Neuroscience, 21, 2451-2461.

Pfurtscheller, G. (2001). Functional brain imaging based on ERD/ERS. Vision Research, 41, 1257-1260.

Pfurtscheller, G., \& Aranibar, A. (1979). Evaluation of eventrelated desynchronization (ERD) preceding and following voluntary self-paced movement. Electroencephalography and Clinical Neurophysiology, 46, 138-146.

Sandstrom, N. J., Kaufman, J., \& Huettel, S. A. (1998). Males and females use different distal cues in a virtual environment navigation task. Brain Research, Cognitive Brain Research, 6, 351-360.

Scherg, M. (1990). Fundamentals of dipole source potential analysis. Advances in Audiology, 6, 40-69.

Seubert, J., Humphreys, G. W., Muller, H. J., \& Gramann, K. (2008). Straight after the turn: The role of the parietal lobes in egocentric space processing. Neurocase, 14, 204-219.

Shelton, A. L., \& Gabrieli, J. D. (2002). Neural correlates of encoding space from route and survey perspectives. Journal of Neuroscience, 22, 2711-2717.

Vogeley, K., \& Fink, G. R. (2003). Neural correlates of the first-person-perspective. Trends in Cognitive Sciences, 7, 38-42

Whitlock, J. R., Sutherland, R. J., Witter, M. P., Moser, M. B., \& Moser, E. I. (2008). Navigating from hippocampus to parietal cortex. Proceedings of the National Academy of Sciences, U.S.A., 105, 14755-14762. 
Wilson, K. D., Woldorff, M. G., \& Mangun, G. R. (2005). Control networks and hemispheric asymmetries in parietal cortex during attentional orienting in different spatial reference frames. Neuroimage, 25, 668-683.

Wipf, D., \& Nagarajan, S. (2009). A unified Bayesian framework for MEG/EEG source imaging. Neuroimage, 44, 947-966.

Wolbers, T., \& Buchel, C. (2005). Dissociable retrosplenial and hippocampal contributions to successful formation of survey representations. Journal of Neuroscience, 25, 3333-3340.
Wolbers, T., Wiener, J. M., Mallot, H. A., \& Buchel, C. (2007). Differential recruitment of the hippocampus, medial prefrontal cortex, and the human motion complex during path integration in humans. Journal of Neuroscience, 27, 9408-9416.

Worden, M. S., Foxe, J. J., Wang, N., \& Simpson, G. V. (2000). Anticipatory biasing of visuospatial attention indexed by retinotopically specific alpha-band electroencephalography increases over occipital cortex. Journal of Neuroscience, 20, RC63. 


\section{AUTHOR QUERIES}

\section{AUTHOR PLEASE ANSWER ALL QUERIES}

During the preparation of your manuscript, the questions listed below arose. Kindly supply the necessary information.

1. The following references were cited in the text but have no matching entries in the reference list: Delorme et al., in preparation; Makeig et al., 2004. Please include in the list or, alternatively, delete the citations from the text.

2. Gramann et al., in press: Please update this reference if it has already been published.

\section{END OF ALL QUERIES}

